Revista de Matemática: Teoría y Aplicaciones 2009 16(1) : 105-126

CIMPA - UCR ISSN: 1409-2433

\title{
LAS ECUACIONES DE REYNOLDS Y LA RELACIÓN DE CLAUSURA
}

\author{
José Roberto Mercado*
}

Recibido/Received: 20 Feb 2008 - Aceptado/Accepted: 19 Dic 2008

\begin{abstract}
Resumen
Nos planteamos el problema de obtener la relación de clausura para las ecuaciones de Reynolds. Y, como objetivo secundario, obtener expresiones analíticas para los esfuerzos de Reynolds; mostrando su salto de discontinuidad, como expresión de la ruptura de la simetría, la que interpretamos como una salto en el índice de ocupación del espacio. Nuestro resultado principal consiste en que el esfuerzo de Reynolds se expresa como la derivada fraccional de la velocidad media, siendo el orden de la derivada el índice de ocupación espacial; lo que transforma la ecuación de Reynolds en una ecuación integro-diferencial. Formulamos un modelo de Prandtl fraccional, en donde la raíz cuadrada del esfuerzo de Reynolds depende de la derivada fraccional de la velocidad media; y se recupera el modelo de Prandtl cuando la derivada fraccional tiende al orden entero de valor unitario. Se presenta una transición regularizante entre la velocidad de la subcapa inercial y la viscosa; y se obtiene la constante de Nikuradse como el equivalente hidráulico de la constante de Euler, que mide la razón de las dos escalas. Analizamos las ecuaciones de Reynolds para un flujo entre dos planos paralelos, o para un tubo, a través de una ecuación de Fokker-Planck estacionaria. Se obtiene el perfil de velocidades tanto para la subcapa viscosa; como para la subcapa inercial. El fluido presenta una transición de segundo orden que se manifiesta, a nivel macro, como un salto de discontinuidad del esfuerzo de Reynolds, en tanto parámetro de orden, con ruptura de la simetría; y a nivel micro, como un salto en el índice de ocupación del espacio.
\end{abstract}

Palabras clave: Ecuaciones y esfuerzos de Reynolds, subcapas viscosa e inercial, modelo de Prandtl, derivada fraccional, problema inverso, ecuación de Camassa-Holm, transiciones de segundo orden, parámetro de orden.

${ }^{*}$ Instituto Mexicano de Tecnología del Agua, IMTA, Paseo Cuauhnáhuac 8532, Progreso, Jiutepec, Mor., C. P. 62550, México; Fax: $+\{52\} 77732936$ 82. E-Mail: rmercado@tlaloc.imta.mx 


\begin{abstract}
We posed the problem to obtain the closure relation for the Reynolds equations. And like secondary target, to obtain analytical expressions for the Reynolds stress. Showing its jump of discontinuity like expression of the rupture of the symmetry; the one is interpret by us as a jump in the index of occupation of the space. Our main result consists of which the Reynolds stress is expressed like the fractional derived one from the average velocity.

Being the order of the derived one index of space occupation; what the Reynolds equations transform into differential integral equations. We formulate a model of fractional Prandtl where the squared root of the Reynolds stress depends of the fractional derived one from the average velocity and the model of Prandtl is recovered when the fractional derived one tends to the whole of value. A regularizated transition appears between velocity of the inertial sub-layer and the viscous and the constant of Nikuradse is obtained like the hydraulic equivalent of the Euler's constant, who measures the reason of the two scales. We analyze the Reynolds equations for a flow between two planes parallels, through an equation of stationary Fokker-Planck. The velocity profile for the viscous sub-layer is obtained as much; like for the inertial sub-layer. The fluid displays a transition of second order that is pronounced, at level macro, as a jump of discontinuity of the Reynolds stress in as much parameter of order, with rupture of the symmetry; and at micro level, as a jump in the index of occupation of the space.
\end{abstract}

Keywords: Reynolds equations and stress, boundary layer, viscous layer, Prandtl's model, fractional derivatives, inverse problem, Camassa-Holm equation, second-order transition, order parameter.

Mathematics Subject Classification: 35Q35, 35Q30.

\title{
1 Introducción
}

El propósito del presente artículo es obtener la relación de clausura para las ecuaciones de Reynolds, lo cual significa una relación entre la velocidad media, temporal, y el esfuerzo de Reynolds. Esta relación transforma a las ecuaciones de Reynolds en ecuaciones integrodiferenciales; y, además, nos permite formular una generalización de la ley de Hooke, como una relación entre la deformación y el esfuerzo.

El propósito secundario es describir la transición del movimiento de un fluido; primero, de turbulento en laminar como una convolución con una función regularizante; y segundo, recíprocamente, describir la transición del movimiento desde laminar a turbulento, como una transición de fase. Como consecuencia se obtienen las expresiones analíticas para los esfuerzos de Reynolds, en el caso de flujo entre placas paralelas; y se observa su salto de discontinuidad bajo un pequeño cambio de la velocidad cerca de su valor de transición como manifestación de una transición de segundo orden, en donde se presenta una ruptura, de la simetría, y entonces, del parámetro de orden; y que interpretamos como un cambio abrupto en el índice de ocupación del espacio.

Los resultados nos muestran que el esfuerzo viscoso es de rango, relativamente, largo; en tanto su contraparte, el inercial, lo vemos como de corto rango. La interpretación la 
encontramos en la ocupación del espacio, para la primera, la ocupación es casi total, por lo que imaginamos, a nivel microscópico, las moléculas del fluido más cerca unas de otras, por lo que las interacciones de dipolo serán más fuertes, o habrá mayor propensión a la formación de los puentes de hidrógeno de cada molécula con cuatro de sus vecinas más próximas; cuando se las compara con la segunda fase, en donde la ocupación del espacio es más parcial, luego las moléculas estarían, relativamente, más lejos unas de otras, dando lugar a una interacción más débil entre los distintos dipolos moleculares.

Aunque podría pensarse que encontrar el perfil de velocidades, para un flujo turbulento estacionario de un fluido incompresible, es un problema sencillo, todavía es un problema no resuelto, [12].

Nuestro punto de partida es la ecuación de Navier-Stokes, la cual reformulamos introduciendo la conservación de la masa expresada en la divergencia nula de la velocidad, debido a la hipótesis de incompresibilidad del fluido. Viendo las variaciones de la velocidad contra la velocidad media temporal como una variable aleatoria, podemos reconocer en las fluctuaciones de la velocidad, un proceso estocástico estacionario, en sentido amplio. Teniendo un modelo para la distribución de probabilidades de las variaciones espaciales de la velocidad, calculamos los promedios temporales sobre la base de la hipótesis ergódica, lo que permite filtrar una gran cantidad de términos y obtener las ecuaciones de Reynolds. Ésta es una ecuación de evolución en donde la función fuente depende de los esfuerzos de Reynolds, los que a su vez dependen de las minúsculas fluctuaciones de la velocidad; y ellos originan la difusión de las diferencias de momentum. Sin embargo, como es conocido, ésta no es una ecuación cerrada, porque no tenemos una dependencia explícita del esfuerzo de Reynolds con la velocidad media, la cual es la variable de campo para esta ecuación diferencial.

Nos encontramos con dos problemas teóricos mayores, por una parte, cerrar las ecuaciones de Reynolds encontrando una relación analítica entre los esfuerzos de Reynolds y la velocidad media. Habíamos pensado que esto nos permitiría manejarlas como ecuaciones diferenciales parciales usuales; sin embargo, el resultado es que surge una ecuación integro-diferencial, pero, de todas maneras, esto nos posibilita el estudiar sus propiedades y soluciones ya sea por la vía de la transformada de Laplace, del análisis, o por métodos numéricos. Por otra parte, contamos con un teorema sobre la existencia de las soluciones de la ecuación de Navier-Stokes, pero sólo limitada a fluidos que tenga una gran viscosidad, lo que permite hacer cumplir la hipótesis de coercividad; nosotros creemos que esta hipótesis se cumple en la porción viscosa del esfuerzo de Reynolds, pero no en la inercial; lo cual se debe, como ya anotamos, a la parcial ocupación del espacio debida a las transiciones de segundo orden. Por tanto, sentimos que si se definen los espacios de funciones que incorporen las medidas dependientes del índice de ocupación se podría intentar demostrar la existencia de las soluciones de la ecuación de Navier-Stokes en las dos fases (de segundo orden).

Obtenemos los perfiles de velocidad que resuelven la ecuación diferencial de Reynolds e identificamos sus condiciones de contorno. Esto da lugar a un punto de bifurcación en donde se cruzan los dos perfiles de velocidad y nos aporta un parámetro espacial del fenómeno, al que se le conoce como espesor de la capa límite; el cual define la frontera interna de la capa límite como la línea de equilibrio entre la acción externa y la interna. 
Ahora podemos definir el esfuerzo de Reynolds en términos de la velocidad, calcular la velocidad de transición y proceder a dar las interpretaciones macro y microscópicas del fenómeno.

Los resultados de este artículo fueron estimulados por la propuesta de W. Chen, (2006)[6], en el sentido de que el esfuerzo de Reynolds sea un Laplaciano fraccional de la velocidad media.

El artículo está organizado de la forma siguiente: en la sección 2, iniciamos el desarrollo del plan trazado en esta introducción; en la subsección 1, comenzamos con la ecuación de Navier-Stokes, luego en la 2, obtenemos las ecuaciones de Reynolds; después en la subsección 3, la particularizamos al caso de un flujo entre placas paralelas, en al subsección 4, estudiamos los elementos para la derivada fraccional. En la sección 3, consideramos la transición de la velocidad de turbulenta a laminar; obteniendo la relación entre las constantes de Nikuradse y la Euler. En la subsección 3.1 obtenemos la relación de clausura. En la 3.2, buscamos los esfuerzos de Reynolds, representando al esfuerzo como derivada fraccional de la velocidad; en la 3.3, estudiamos los perfiles de velocidad y el punto de bifurcación; y, formulamos el modelo de Prandtl fraccional; en la 3.4 aplicamos los resultados a la ecuación Camassa-Holm para canales. En la sección 4 analizamos la transición de fase y terminamos encontrando el esfuerzo de Reynolds, con su salto de discontinuidad a la velocidad de transición. En la sección 5, enunciamos las conclusiones, y en la sección última, presentamos las principales referencias que nos han servido de apoyo.

\section{Desarrollo}

\subsection{Ecuación de Navier-Stokes}

La ecuación de Navier-Stokes surge de aplicar la segunda ley de Newton, o ley de la fuerza, a un elemento de masa sometido a las interacciones de las fuerzas de esfuerzos, como la presión, y a las fuerzas de cuerpo, como la fricción y la gravedad; junto con la condición de la conservación de la masa, que debido a la característica de la incompresibilidad del fluido, se convierte en la ausencia de divergencia. Además, se debe agregar la condición inicial para la velocidad del fluido. En la ecuación (1), $v$ es la velocidad instantánea, $p$ la presión del fluido, $\nu$ la viscosidad cinemática, $\rho$ la densidad del fluido, $f$ una fuerza de cuerpo, $t$ el tiempo, $\nabla$ y $\Delta$, son los operadores gradiente y Laplaciano respectivamente [9], [30],

$$
\frac{\partial}{\partial t} \mathbf{v}+(\mathbf{v} \cdot \nabla) \mathbf{v}=\nu \Delta \mathbf{v}-\operatorname{grad} \frac{\mathbf{p}}{\rho}+f, \nabla \cdot \mathbf{v}=0
$$

Para reformular la ecuación diferencial, incorporamos la divergencia nula, multiplicamos por la densidad del fluido, y obtenemos (2); en donde $v_{i}$ son las distintas componentes espaciales de la velocidad; y, hemos usado la convención de repetición de índices para indicar la suma sobre ellos,

$$
\rho \frac{\partial}{\partial t} v_{i}+\rho \frac{\partial}{\partial x_{j}}\left(v_{i} v_{j}\right)=-\frac{\partial}{\partial x_{i}} p+\rho \nu \Delta v_{i}
$$




\subsection{Ecuaciones de Reynolds}

Las variaciones de la velocidad pueden concebirse como las desviaciones de la velocidad con respecto a su valor medio temporal; de tal manera que las variaciones de la velocidad se vean como una variable aleatoria de media nula. En tanto la presión, también, puede descomponerse en una forma similar, ecuación (3). Esta variable aleatoria es estacionaria, en amplio sentido, porque su media o esperanza matemática es constante, independiente de su parámetro índice, el tiempo; y la función de correlación depende sólo de las variaciones de este parámetro,

$$
\delta v_{i}=\bar{v}_{i}-v_{i}, \delta p_{i}=\bar{p}_{i}-p_{i}
$$

Sabemos que la distribución de las variaciones de la velocidad obedece a una distribución Beta, con exponentes de forma que dependen del índice de ocupación del espacio, definido por el cociente entre la dimensión fractal y la topológica; el cual, a su vez, depende del índice de estabilidad Lévy; o equivalentemente, la distribución de las diferencias de la velocidad es la transformada de Laplace inversa de una distribución Lévy. Por otra parte, la hipótesis ergódica significa la posibilidad de igualar el promedio temporal de las velocidades con el promedio probabilístico, calculado sobre la base del conocimiento de la distribución, el cual es conocido también como promedio de ensamble, [17].

Por tanto, cuando la velocidad y la presión se descomponen en la media más las desviaciones, como en (4),

$$
v_{i}=\bar{v}_{i}+\delta v_{i}, p_{i}=\bar{p}_{i}+\delta p_{i}
$$

para luego expandir la ecuación de Navier -Stokes, y tamizarla con el promedio temporal sobre la base de la hipótesis ergódica, entonces se obtienen las ecuaciones de Reynolds (5), $[6]$,

$$
\rho\left(\frac{\partial}{\partial t} \bar{v}_{i}+\frac{\partial}{\partial x_{j}}\left\langle\bar{v}_{i} \bar{v}_{j}\right\rangle\right)=-\frac{\partial}{\partial x_{j}} \bar{p}+\rho \nu \frac{\partial^{2}}{\partial x_{j}^{2}} \bar{v}_{i}-\rho \frac{\partial}{\partial x_{j}}\left\langle\delta v_{i} \delta v_{j}\right\rangle .
$$

\subsection{Flujo entre placas paralelas}

Para el caso de un flujo entre placas paralelas, se hacen más explícitas las ecuaciones de Reynolds junto con sus condiciones de contorno de no-deslizamiento sobre las placas, suponiéndolas lisas.

La ecuación resultante puede analizarse a través de una ecuación de Fokker-Planck estacionaria con una fuente que depende sólo de la variable de campo, la velocidad media del flujo, con coeficiente advectivo nulo y coeficiente de difusión constante igual a la viscosidad del fluido, [21].

El tensor de Reynolds actúa como generador de la difusión del momentum ligado a las variaciones espaciales de la velocidad media, ecuación (6), [25],

$$
\rho \frac{\partial}{\partial x_{j}}\left\langle\delta v_{i} \delta v_{j}\right\rangle=-\frac{\partial}{\partial x_{j}} \bar{p}+\rho \nu \frac{\partial^{2}}{\partial x_{j}^{2}} \bar{v}_{i}
$$




\subsection{Método: derivada fraccional}

Se sabe que Leibniz se planteó el problema de la derivada de orden $\frac{1}{2}$, pero como una curiosidad matemática; sin embargo, fue Liouville quien primero abordó, en 1832, de forma consistente el operador integral que hoy conduce a la derivada fraccional.

Podemos introducir la integral y la derivada fraccional a partir del problema de Abel de la tautocrona (igual tiempo, 1826). Pero, el importante problema de la tomografía computarizada CT, 1971, también conduce a una transformación integral de Abel.

Los dos problemas se traducen en encontrar la función que resuelva un producto de convolución, como en (12). Una generalización del problema de Abel consiste en encontrar la función que permita la transformación de una potencia en otra potencia, tal como lo hacen la derivada y la integral, de orden entero, cuando actúan sobre una potencia. La solución se busca en el ámbito de las ecuaciones integrales de Volterra de primera especie.

En [19] se consideran las medidas de Hausdorff sobre los conjuntos de Baire, que luego se extienden a los conjuntos de Borel; de tal manera que existe una única medida de Baire, definida en el álgebra de conjuntos adecuada, que permite definir una funcional lineal, con la extensión y renormalización apropiada, y que puede considerarse como la medida de Hausdorff. En [28] la funcional lineal se da por una integral en donde la distribución de Hadamard es evaluada sobre funciones de prueba localmente integrables; o es la transformada de Mellin de funciones seccionadas por la distribución de Heaviside. Por tanto, la derivada fraccional nos pone en contacto con los fractales, ya que la distribución de Hadamard se construye sobre la base de una generalización del proceso de Cantor.

Por ello, queremos recordar que los fractales autosimilares pueden introducirse a partir del conjunto de Cantor, el cual se elabora sobre la base de dos parámetros $p=2$ y $q=3$, los rasgos y la resolución; de suerte que con el primero se crea una sucesión que crece como una integral de orden entero $2^{n}$; en tanto que con el segundo se construye una sucesión que decrece como una derivada de orden entero $(1 / 3)^{n}$; siendo $1 / 3$ el valor máximo posible para el parámetro de la resolución.

El proceso puede generalizarse para un par de parámetros $p$ y $q$, mayores que 1 . En particular, el núcleo o distribución de Hadamard $\frac{x^{\nu-1}}{\Gamma(\nu)}$ se obtiene de un proceso de Cantor generalizado, similar a la construcción de los fractales auto-similares, en donde la función de la resolución se la define por $f(q)=q^{-\nu}, \nu>0, q>1$; y el proceso se inicia con el cálculo

$$
H_{\nu, n}=\left.\left((-1)^{n} D_{q}^{n}\left(q^{-\nu}\right)\right) \cdot(n+1) D_{p}^{(-n)}(p)\right|_{q}
$$


Con lo que se obtiene:

$$
\begin{aligned}
H_{\nu, 1}= & \nu q^{-\nu-1} \cdot \frac{q^{2}}{1}=\frac{(\nu)_{1}}{1} q^{-\nu+1} \\
H_{\nu, 2}= & \nu(\nu+1) q^{-\nu-2} \cdot \frac{q^{3}}{2}=\frac{(\nu)_{2}}{2} q^{-\nu+1} \\
H_{\nu, 3}= & \nu(\nu+1)(\nu+2) q^{-\nu-3} \cdot \frac{q^{4}}{3 !}=\frac{(\nu)_{3}}{3 !} q^{-\nu+1} \\
& \vdots \\
H_{\nu, n}= & \nu(\nu+1)(\nu+2) \cdots(\nu+n-1) q^{-\nu-n} \cdot \frac{q^{n+1}}{n !}=\frac{(\nu)_{n}}{n !} q^{-\nu+1}
\end{aligned}
$$

en donde $(\nu)_{n}=\nu(\nu+1) \cdots(\nu+n-1)=\frac{\Gamma(\nu+n)}{\Gamma(\nu)},(\nu)_{0}=1,(1)_{n}=n$ !, son los símbolos de Pochhammer, expresados como productos sucesivos y en términos de la función gamma de Euler, [2].

Se discretiza la sucesión de las resoluciones como $q_{n}=\frac{n}{x}, x>0 ; \mathrm{y}$ con $\Gamma_{n}(\nu)=$ $\frac{(1)_{n}}{(\nu)_{n+1}} n^{\nu}$, como la sucesión que converge, con $n$, a la función Gamma de Euler; y se obtiene en el límite

$$
H_{n}=\frac{x^{\nu-1}}{\Gamma_{n}(\nu)\left(\frac{\nu}{n}+1\right)} \rightarrow \frac{x^{\nu-1}}{\Gamma(\nu)} .
$$

Este resultado puede también enunciarse diciendo que la transformada de Laplace inversa, de la función completamente monótona $q^{-\nu}$, es la función de crecimiento exponencial $\frac{x^{\nu-1}}{\Gamma(\nu)}$, según la fórmula de Post; pero que además permite extender el dominio desde $q>1$ hasta $q>0$,

$$
L^{-1}\left(q^{-\nu}\right)(x)=\frac{x^{\nu-1}}{\Gamma(\nu)} .
$$

Desde la perspectiva de las distribuciones, se dice que este núcleo es una distribución temperada con capacidad para actuar sobre las funciones de prueba tomadas del conjunto de las funciones locamente integrables, y en el rango $\operatorname{Re}(\nu)>0$, [10].

Integrando por partes, se obtiene una representación para la distribución como derivada de orden entero y su reducción a la distribución de Dirac, $\forall n \in \mathbb{N}$,

$$
\frac{x^{\nu-1}}{\Gamma(\nu)}=\left.D_{x}^{n}\left(\frac{x^{\nu-1+n}}{\Gamma(\nu+n)}\right) \rightarrow \frac{x^{\nu-1}}{\Gamma(\nu)}\right|_{\nu=0}=\delta_{0}(x),
$$

Pero además, las distribuciones de Hadamard, constituyen un semigrupo bajo el producto de convolución y teniendo como su unidad a la distribución de Dirac

$$
H_{\nu_{1}} * H_{\nu_{2}}=H_{\nu_{1}+\nu_{2}}, \quad H_{0}=\delta_{0}, \quad \operatorname{Re}\left(\nu_{i}\right)>0 .
$$

Con base en este semigrupo se define la integral fraccional por la convolución, [27], [15],

$$
{ }_{0} D^{-\nu} \varphi(x)=D^{-\nu} \varphi(x)=H_{\nu} * \varphi \text {. }
$$


Por tanto, con el proceso de Cantor generalizado sobre las funciones en potencia, completamente monótonas, se obtienen las distribuciones del semigrupo de Hadamard; las que actuando como núcleos definen la integral fraccional.

En una forma análoga, con un proceso de Cantor generalizado construido a partir de la función completamente monótona $f(q)=\frac{\ln q}{q}$; produce la sucesión $P_{n}=-\ln e^{\gamma_{n}} x$; que en el límite conduce a $P=-\ln e^{\gamma} x$; en donde $\gamma_{n}$ es la sucesión $\gamma_{n}=\sum_{j=1}^{n} \frac{1}{j}-\ln n$, que converge a la constante de Euler $\gamma$. Por lo que el proceso puede describirse por la igualdad $-\ln e^{\gamma} x=L^{-1}\left(\frac{\ln q}{q}\right)(x)$; y con la propiedad de semejanza para la transformada de Laplace, $L^{-1}\left(f\left(\frac{s}{\lambda}\right)\right)(x)=|\lambda|\left(L^{-1} f(s)\right)(\lambda x)[2]$, [13], se transforma en

$$
\ln x=L^{-1}\left(-\frac{\ln e^{\gamma} q}{q}\right)(x) .
$$

Que representa al logaritmo como una función de crecimiento exponencial; con el parámetro de resolución como la variable conjugada a la posición, según la transformada de Laplace; y a la constante de Euler como el factor de escala.

\section{La transición en la velocidad}

La primera descripción de la transición nos conduce a planteamos el problema de la transformación de la velocidad en la subcapa inercial a la misma, en la subcapa viscosa. Por lo que, en principio, se supone que tenemos la velocidad media temporal como solución de las ecuaciones de Reynolds, regular en el tiempo, irregular en el espacio; y se la quiere regularizar promediando a través de una convolución que realice la transición,

$$
\langle v\rangle_{t} * \rho(y)=\frac{u_{*}^{2}}{\nu} y \mapsto\left\langle\frac{v}{u_{*}}\right\rangle * \rho(y)=\frac{u_{*}}{\nu} y ;
$$

en (14), $v$ denota la velocidad; $\rho$ la función regularizante; $y$ la coordenada transversal; $u_{*}$ la velocidad de corte; $\nu$ la viscosidad cinemática; $l_{0}=\nu / u_{*}$ la longitud intrínseca al fenómeno; y el paréntesis angular, y su subíndice, están simbolizando el promedio temporal.

La transformación de renormalización se representa a través de los parámetros de escala dados por $\lambda_{1} \mathrm{y} \lambda_{2}$, proporcionales a la longitud intrínseca $l_{0}$, como en la ecuación (15); la coordenada transversal por $x$; la variable de integración por $t$; la constante de Kármán por $\kappa$; la coordenada transversal para la condición de frontera del perfil logarítmico por $y^{\prime}$; y la constante para el factor de estabilidad por $C,[24]$. Entonces, la convolución de la ecuación (14) la podemos enunciar como:

$$
\int_{0}^{x} \ln \frac{(x-t)}{\lambda_{2}} \rho(t) d t=\frac{x}{\lambda_{1}}, \lambda_{2}=y^{\prime}=C l_{0}, \lambda_{1}=l=\kappa l_{0}, l_{0}=\frac{\nu}{u_{*}} ;
$$

Por tanto, se plantea el problema inverso contenido en la ecuación integral (15); en donde se debe encontrar la función regularizante $\rho$. Por lo que se trata de invertir la transformada integral, en tanto integral de Volterra dependiente de una convolución, [13], [7]. 
Se requiere la transformada de Laplace del logaritmo, y ésta se obtiene de la relación dada por la ecuación (13), en donde se recupera el logaritmo como transformada inversa de Laplace de una función completamente monótona

$$
\ln x=L^{-1}\left(-\frac{\ln e^{\gamma} s}{s}\right)(x) .
$$

Por otra parte, cambiando en la funcional de Hadamard $\nu \mapsto \nu-1$, para luego integrar con respecto a $\nu$, intercambiando la integral con la transformada inversa, se tiene

$$
\int_{0}^{\infty} \frac{x^{\nu}}{\Gamma(\nu+1)} d \nu=L^{-1}\left(\frac{1}{s \ln s}\right), \operatorname{Re}(\nu)>0 .
$$

Ahora, con el objetivo de introducir los factores de escala de la transformación de renormalización, volvemos a usar la propiedad de semejanza de la transformada de Laplace, en la forma $L\left(F\left(\frac{x}{\lambda}\right)\right)(s)=|\lambda|(L F(x))(\lambda s)$,

$$
\int_{0}^{\infty} \frac{\left(\frac{1}{\lambda_{2} e^{\gamma}} x\right)^{\nu}}{\Gamma(\nu+1)} d \nu=L^{-1}\left(\lambda_{2} e^{\gamma} \frac{1}{\left(\lambda_{2} e^{\gamma} s\right) \ln \left(\lambda_{2} e^{\gamma} s\right)}\right)=L^{-1}\left(\frac{1}{s \ln \left(\lambda_{2} e^{\gamma} s\right)}\right) .
$$

Por la relación dual entre una función de crecimiento exponencial y su transformada de Laplace, que convierte un producto de convolución en un producto de transformadas; y con la propiedad de semejanza, se obtiene (19), en donde la tilde se utiliza para simbolizar la transformada de Laplace,

$$
\lambda_{2}(\tilde{\ln })\left(\lambda_{2} s\right) \cdot \tilde{\rho}(s)=\frac{\tilde{x}(s)}{\lambda_{1}} .
$$

Entonces, llegamos a

$$
\tilde{\rho}(s)=\frac{\frac{1}{\lambda_{1}} \tilde{x}(s)}{\lambda_{2}(\widetilde{\ln })\left(\lambda_{2} s\right)}=\frac{\frac{1}{\lambda_{1}} \frac{\Gamma(2)}{s^{2}}}{\lambda_{2} \frac{1}{\lambda_{2} s}\left(-\ln \lambda_{2} e^{\gamma} s\right)}=-\frac{\frac{1}{\lambda_{1}}}{s\left(\ln \lambda_{2} e^{\gamma} s\right)} .
$$

Por lo que, finalmente, se obtiene la solución de la ecuación integral que transforma el perfil inercial en el perfil laminar

$$
\rho(x)=-\int_{0}^{\infty} \frac{1}{\lambda_{1}} \frac{\left(\frac{1}{\lambda_{2} e^{\gamma}} x\right)^{\nu}}{\Gamma(\nu+1)} d \nu
$$

en donde puede observarse la superposición de los núcleos, que constituyen las funcionales de Hadamard, y que definen la integral fraccional que se obtuvieron inicialmente a partir de un proceso de Cantor generalizado (8),

$$
d \mu\left(\nu, x, \lambda_{1}, \lambda_{2}\right)=\frac{1}{\lambda_{1}} \frac{1}{\left(\lambda_{2} e^{\gamma}\right)^{\nu}} \frac{x^{\nu}}{\Gamma(\nu+1)} .
$$

Si se substituye $\nu \mapsto \nu-1$, y se insertan los parámetros hidráulicos de escala de la ecuación (15), resulta que la razón de estas escalas depende sólo del parámetro de Nikuradse; 
$\frac{\lambda_{1}}{\lambda_{2}}=\frac{\kappa}{C}=N$; por lo que, tomando en cuenta la representación de la distribución de Dirac de la ecuación (10), resulta el límite débil

$$
\frac{1}{\lambda_{1}} \frac{1}{\left(\lambda_{2} e^{\gamma}\right)^{\nu-1}} \frac{x^{\nu-1}}{\Gamma(\nu)} \stackrel{\nu \rightarrow 0}{\rightarrow} \frac{\lambda_{2}}{\lambda_{1}} e^{\gamma} \delta_{0}(x)=\frac{e^{\gamma}}{N} \delta_{0}(x) .
$$

De tal manera que si se define otra transformación de renormalización sobre la base de los cambios de escala dados por $\lambda_{i}=\frac{\lambda_{i}^{\prime}}{a_{i}}, i \in\{1,2\}$; se obtiene la razón de las constantes como $\frac{e^{\gamma}}{N}=\frac{\lambda_{2}^{\prime}}{\lambda_{1}^{\prime}}, \nu \rightarrow 0$.

\subsection{Relación de clausura}

Los resultados de la subsección anterior y los reportados en [17], nos inducen a replantearnos la ecuación de Reynolds unidimensional, en donde el exponente del término difusivo tenga un valor máximo cercano a 2, característico de la distribución gaussiana; por lo que consideramos que asume la forma (24),

$$
c_{0}=c_{1} \rho \nu D_{y}^{1+\beta} u+D_{y}^{1} \tau, \beta=\frac{D_{f}}{D_{T}}
$$

Luego entonces, si la velocidad media la representamos por una integral fraccional del esfuerzo, con una constante que ajuste las unidades $u=-c_{2} D_{y}^{-\gamma} \tau$; y ésta las sustituimos en la ecuación de Reynolds (24), tenemos,

$$
0=\left(-c_{1} \rho \nu c_{2} D_{y}^{1+\beta-\gamma} \tau+D_{y}^{1} \tau\right)=D_{y}^{1}\left(-c_{1} \rho \nu c_{2} D_{y}^{\beta-\gamma}+1\right) \tau .
$$

Entonces, si escogemos $\gamma=\beta$ y $c_{2}=1 / c_{1} \rho \nu$, obtenemos (25)

$$
u=-\frac{1}{c_{1} \rho \nu} D^{-\beta} \tau, \beta=\frac{D_{f}}{D_{T}}
$$

Y por tanto la relación de clausura es

$$
\tau=-c_{1} \rho \nu D^{\beta} u
$$

En (24), puede absorberse a $c_{0}=\frac{d P}{d x}=-\frac{\rho u_{*}^{2}}{y_{0}}=-\frac{\tau_{0}}{y_{0}}$, escribiendo

$$
0=c_{1} \rho \nu D_{y}^{1+\beta} u+D_{y}^{1}\left(\tau+c_{0} y+c t e\right), \bar{\tau}=\tau+c_{0} y+c t e,
$$

Por lo que se tiene otra normalización del esfuerzo, de suerte que sobre la pared el esfuerzo tienda a 0 , y en la línea media vuelva a ser nulo; entonces, haciendo $\bar{\tau}-\tau=\tau_{0}\left(1-\frac{y}{y_{0}}\right)$; y $u^{+}=\frac{u}{u_{*}}, y^{+}=\frac{y}{l_{0}}, l_{0}=\frac{\nu}{u_{*}} ;$ la relación de clausura será [31],

$$
\frac{\bar{\tau}}{\tau_{0}}=\left(1-\frac{y}{y_{0}}\right)+D_{y^{+}}^{\beta} u^{+}
$$


Por tanto, el esfuerzo de Reynolds es proporcional a la derivada fraccional de la velocidad media; siendo el orden de la derivada el índice de ocupación espacial, el cual se expresa como el cociente entre la dimensión fractal y la dimensión topológica. Y, dualmente, la velocidad media resulta proporcional a la integral fraccional del esfuerzo de Reynolds. De tal manera, que si este índice se acerca a la unidad el esfuerzo se expresaría como cercano a la derivada de la velocidad media, pero esto se conseguiría sólo en forma extrapolada; mientras, en las situaciones más comunes, se situarían en la semiderivada, derivada fraccional del orden cercano a $\frac{1}{2}$, como en el problema de Abel y la tomografía CT; de acuerdo con los resultados reportados en [17].

En particular, para el caso elástico, se obtiene una generalización de la Ley de Hooke, en donde la deformación es proporcional a la integral fraccional del esfuerzo. Y, cuando el índice de ocupación tiende a cero, se produce la proporcionalidad entre el esfuerzo y la deformación, lo que recupera la Ley de Hooke,

$$
u=c D^{-\beta} \tau, \beta \rightarrow 0 .
$$

\subsection{Esfuerzos de Reynolds}

Los resultados de la sección anterior pueden aplicarse inmediatamente al caso del flujo entre caras paralelas; uno de los pocos casos en los que contamos con expresiones analíticas para el esfuerzo, como las obtenidas en [18]; lo que nos permitirá valorar nuestros resultados. Aunque también, y como segundo caso, los aplicamos a la ecuación de Camassa-Holm [5].

El esfuerzo se expresa en la forma de una potencia de la coordenada transversal, como se muestra en la ecuación (28). Lo que resultó de la forma en potencia para la función de reacción como solución a un problema inverso en [21],

$$
\tau=c y^{a} \mapsto u=D_{y}^{-\beta} \tau=c \frac{\Gamma(a+1)}{\Gamma(a+1+\beta)} y^{a+\beta} .
$$

Para el régimen laminar, de la subcapa viscosa, se conoce que la velocidad media presenta una forma parabólica por lo cual la potencia de la ecuación debe ser 2, luego el exponente

del esfuerzo de Reynolds es $a=2-\beta=2-\frac{D_{f}}{D_{T}}$, si tomamos el límite cuando tiende a 1 , resulta que $a \rightarrow 1$, lo cual es compatible con la descripción obtenida en [18], en donde, para el régimen laminar se obtiene $\alpha-1 \rightarrow 2-1=1$. Si de la descripción no local para la derivada fraccional pasamos a su forma local, lo que se logra definiendo la derivada por la derecha y por la izquierda, para el caso de orden entero, podemos incorporar las condiciones de frontera y se tiene la ecuación (29). Aquí $u_{*}$ es la velocidad de corte o de fricción, $\rho$ la densidad, $\nu$ la viscosidad cinemática, $y_{0}$ la mitad de la separación entre las placas, [1],

$$
\tau(u(y))=-\rho u_{*}^{2} y^{2-\beta} \mapsto \tau(u(y))=-\tau_{0}\left|1-\frac{y}{y_{0}}\right|^{2-\beta},\left.\nu \frac{\partial}{\partial y} u\right|_{y=0}=u_{*}^{2}=\frac{\tau_{0}}{\rho} .
$$

Para la subcapa inercial, debido a los resultados presentados en [18], en donde el esfuerzo de Reynolds (31), resultó ser inversamente proporcional a la coordenada transversal, nos 
induce a plantearnos nuevamente al esfuerzo como una potencia de la coordenada transversal, potencia que debemos encontrar; luego,

$$
\tau=c_{2} y^{b} \mapsto D u=D_{y}^{1-\beta} c_{2} y^{b}=c_{2} \frac{\Gamma(b+1)}{\Gamma(b+\beta)} y^{b-1+\beta}
$$

Y para que sea compatible con [18], debe ser $b+\beta-1=1$; y se obtiene que $b=-\beta$. En particular, si hacemos que $\beta \rightarrow 1$, resulta $b \rightarrow-1$, lo cual coincide con los resultados mencionados.

Pasando de la formulación no local, a la local para la derivada fraccional, podemos incorporar las condiciones de frontera, $\operatorname{con} l=\lambda_{1}$,

$$
\tau(u(y))=D_{y}^{\beta} c y^{-1+\beta} \mapsto \tau(u(y))=-\tau_{0} \frac{l}{y_{0}}\left|\frac{y_{0}}{y}-1\right| .
$$

Si tomamos el límite cuando $\beta$ tiende a 1 , el valor de $b$ tiende a cero, pero se cumple todavía la fórmula (30) la cual es válida en el rango $b>-1 ; \mathrm{y}$, además, resulta ser la derivada fraccional de la unidad, la cual es inversamente proporcional a $y$; y es, todavía, compatible con (31).

El esfuerzo de Reynolds depende del espesor de la capa límite, porque la condición de frontera para la velocidad, en su región inercial, es proporcional a este espesor $y^{\prime}=\frac{1}{N} l$. Y la proporción entre estos dos espesores es la constante de Nikuradse; como razón que es entre las dos escalas, la laminar sobre la inercial $\frac{l}{y^{\prime}}=N=\frac{\lambda_{1}}{\lambda_{2}}$; siendo $l>y^{\prime} ; \mathrm{y}$, según los resultados experimentales, puede ser del orden de cien veces mayor, [24].

Con la normalización de la ecuación (27), y con $y^{+}=\frac{y}{y_{0}} ; l=\kappa l_{0} ; \mathrm{y} l_{0}=\frac{\nu}{u_{*}}$, el esfuerzo de Reynolds contra la coordenada transversal adimensional es

$$
\frac{\bar{\tau}}{\tau_{0}}=\left(1-y^{+}\right)-\kappa \frac{l_{0}}{y_{0}}\left|\frac{1}{y^{+}}-1\right|
$$

Por otra parte, los valores reportados, para distintos números de Reynolds, en [31], son resultados experimentales que se extienden desde 1944 hasta 1984, y se muestran en la tabla siguiente en su forma ascendente. Como las longitudes que dan el espesor de la capa límite se comportan como funciones decrecientes con el crecimiento del número de Reynolds, este comportamiento en escalas relativas se ilustra en la 4ra y 5ta columna, escogiendo, entre los datos disponibles, la ligada al menor número de Reynolds como la base; y observando su variación relativa en la forma $l_{0 i}=l_{0}\left(\frac{R_{e 0}}{R_{e i}}\right)^{a}$, para dos posibles exponentes aportados por los modelos de flujo de Blasius y Hagen-Poiseuille, [24]. Para los valores numéricos: $y_{0}=90 \mathrm{~cm} ; u_{*}=0.3785 \mathrm{~cm} / \mathrm{seg} ; \nu=1.39 \times 10^{-2} \mathrm{~cm}^{2} / \mathrm{seg} ; l_{0}=$ $1.1613 \times 10^{-1.5} \mathrm{~cm}$, se construye la tabla siguiente; para luego elaborar las gráficas de los distintos esfuerzos de acuerdo con el resultado de la ecuación (32) y presentarla en la figura 1. 


\begin{tabular}{l|llll}
\hline \hline & $R_{e}$ & $\frac{R_{e i}}{R_{e 0}}$ & $l_{0 i},(a=1 / 5)$ & $l_{0 i},(a=1 / 2)$ \\
\hline Eckelmann (1944) & 2800 & $=1$ & $1.1613 \times 10^{-1.5}$ & \\
Eckelmann (1944) & 4100 & $\sim 1.5$ & $3.4027 \times 10^{-2}$ & $3.0348 \times 10^{-2}$ \\
Johansson (1984) & 7500 & $\sim 2.7$ & $3.0155 \times 10^{-2}$ & $2.2438 \times 10^{-2}$ \\
Kastrianis \& Eckelmann (1983) & 12600 & $\sim 4.5$ & $2.7183 \times 10^{-2}$ & $1.7311 \times 10^{-2}$ \\
Kastrianis \& Eckelmann (1965) & 57000 & $\sim 20$ & .0201 & $8.1394 \times 10^{-3}$ \\
Comte-Bellot (1965) & 230000 & $\sim 82$ & $1.5206 \times 10^{-2}$ & $4.0521 \times 10^{-3}$ \\
\hline \hline
\end{tabular}

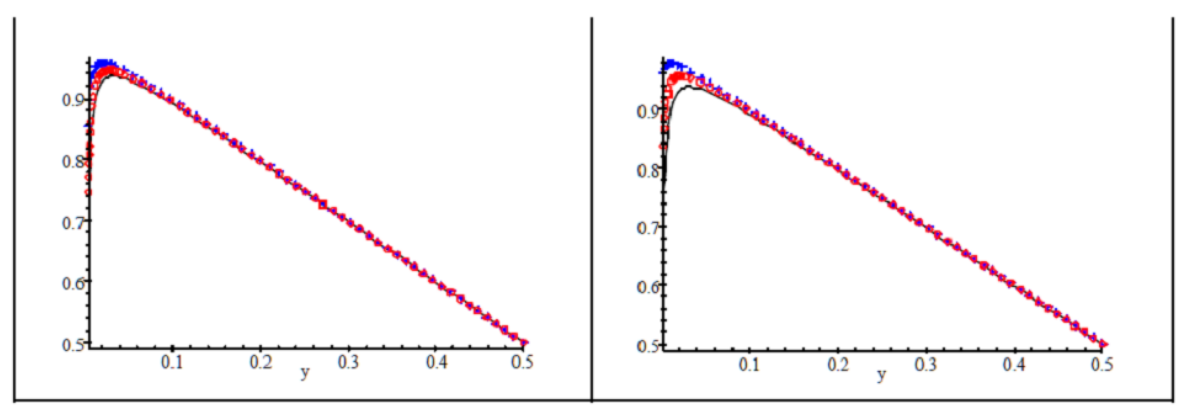

Figura 1: Esfuerzos.

Las gráficas, en la figura 1, muestran, cualitativamente, los mismos comportamientos que exhiben los resultados experimentales presentados en [31]; en donde, con el crecimiento de la coordenada transversal se presenta un crecimiento del esfuerzo hasta llegar a un máximo, para luego decaer, casi linealmente, hasta la línea media, en donde se hace cero, para luego, y en forma simétrica, crecer hasta un máximo y luego decaer nuevamente a cero, casi en la otra placa; fenómeno conocido como la undulación del esfuerzo. Pero además, se observa que entre mayor es el número de Reynolds, mayor es el máximo y que éste se desplaza progresivamente hacia coordenadas menores.

\subsection{Perfiles de velocidad}

El perfil de velocidad, compatible con el esfuerzo de Reynolds viscoso, tiene la forma de potencia, y se muestra en la ecuación (33). Pasando de la formulación no local a la local para la derivada fraccional, podemos incorporar las condiciones de frontera,

$$
u(y)=u_{*} \frac{1}{(3-\beta)} \frac{y_{0}}{l_{0}}\left(1-\left|1-\frac{y}{y_{0}}\right|^{3-\beta}\right),
$$

El régimen laminar se encuentra dentro de la subcapa límite viscosa, y si el índice de ocupación espacial tiende a 1 , los resultados, tanto experimentales como analíticos para 
este régimen, son suficientemente conocidos y muestran que el perfil de velocidades es parabólico. Por tanto, el exponente del perfil de velocidades es $3-\beta \rightarrow 2^{+}$. Y, como se muestra en (34), el esfuerzo de Reynolds varía con la raíz cuadrada de la velocidad, o es lineal con la coordenada transversal, [26], [14].

El perfil de velocidades en la subcapa inercial se muestran en la ecuación (34), en donde las constantes tienen el valor $\kappa=2.5$, y $C=0.0234$. Así que, el perfil de velocidades varía en forma logarítmica con la coordenada transversal; y se ilustra en la figura 2 ,

$$
\frac{u}{u_{*}}=\kappa \ln \frac{y}{C l_{0}}, l_{0}=\frac{\nu}{u_{*}}
$$

Para los valores numéricos: $y_{0}=90 \mathrm{~cm} ; u_{*}=12 \mathrm{~cm} / \mathrm{seg} ; \nu=1.39 \times 10^{-2} \mathrm{~cm}^{2} / \mathrm{seg}$; $l_{0}=1.1583 \times 10^{-3} \mathrm{~cm},[24]$, se obtiene que el punto de corte de los perfiles de velocidad ocurre para el valor $l=0.013605 \mathrm{~cm}$, el cual debe entenderse como el espesor de la capa límite. En tanto, la velocidad de transición, que es la velocidad en el punto de bifurcación, resulta ser, aproximadamente, $0.013605 \times 10334=140.59 \mathrm{~cm} / \mathrm{seg}$. Mostramos el punto de bifurcación y la velocidad de transición en la figura 3 .

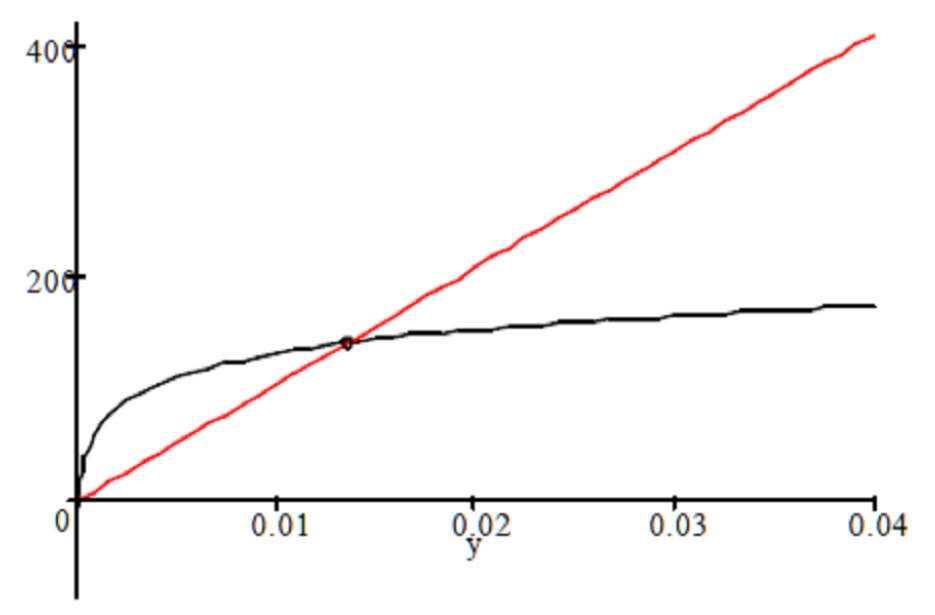

Figura 2: Perfiles de velocidad.

Por lo tanto, al considerar la teoría de capa límite de Prandtl, se colige la existencia de un punto de bifurcación $(l, u(l))$ en el perfil de velocidades; debido a que, en todo entorno de ese punto, se encuentra la solución de la subcapa viscosa, y la logarítmica de la inercial, en donde $l$ es el espesor de la capa límite.

Para la subcapa límite viscosa, la representación del esfuerzo con respecto a la velocidad se da de manera paramétrica, usando la coordenada transversal y como parámetro. Sustituyendo el parámetro $y$, se encuentra la relación entre esfuerzo y velocidad medía, 
$(35)$,

$$
\tau(u)=-\tau_{0}\left|1-2 \frac{l_{0}}{y_{0}} \frac{u}{u_{*}}\right|^{\frac{1}{2}} .
$$

La gráfica del esfuerzo (35) se muestra en la figura 3; siendo los valores numéricos: $y_{0}=$ $90 \mathrm{~cm}, u_{*}=12 \mathrm{~cm} / \mathrm{seg}, \nu=1.39 \times 10^{-2} \mathrm{~cm}^{2} / \mathrm{seg}$; y con el exponente $3-\beta \rightarrow 2^{+}$; [24].

En tanto, para la subcapa inercial, eliminando el parámetro, la coordenada transversal $y$, se obtiene la relación entre el esfuerzo y la velocidad media, (36). Luego, el perfil logarítmico se corresponde con un esfuerzo inercial, en magnitud, decreciente exponencialmente con la velocidad,

$$
\tau(u)=-\tau_{0}\left|N e^{-\frac{u}{\kappa u_{*}}}-\frac{l}{y_{0}}\right| .
$$

Como se observa en la figura 3, el esfuerzo viscoso en magnitud es una función que decrece con el crecimiento de la velocidad media, y por tanto, decrece, también, con la distancia desde las placas; siendo, además, cóncavo; con el espesor de la capa límite representando, aproximadamente, la cota superior de su rango de validez.

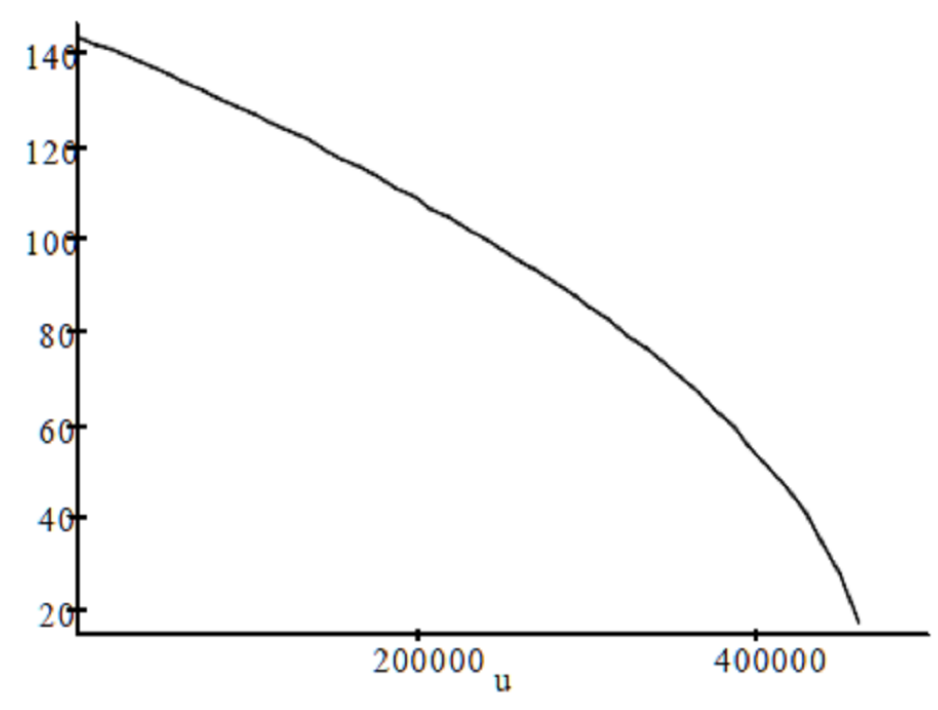

Figura 3: Esfuerzo viscoso.

Por otra parte, en el modelo de Prandtl, la magnitud del esfuerzo es el producto del cuadrado de la longitud de mezcla por el cuadrado del gradiente de la velocidad. Pero la longitud de mezcla también es proporcional a la distancia transversal,

$$
\tau^{\frac{1}{2}}=l_{M} D_{y} u, \quad l_{M}=\kappa^{-1} y .
$$

Luego, si asumimos al esfuerzo como una potencia de la coordenada transversal, como lo hicimos con anterioridad en (28), se tiene

$$
\tau=c_{2} y^{a} \mapsto \kappa c_{2}^{1 / 2} y^{\frac{a}{2}-1}=D_{y} u .
$$


Entonces $\frac{a}{2}-1=-1$, luego $a=0$; por tanto, el esfuerzo es constante. Sin embargo, si generalizamos el modelo de Prandtl introduciendo en consideración un gradiente fraccional; y sustituimos (38) por

$$
\kappa c_{2}^{1 / 2} y^{\frac{a}{2}-1}=D_{y}^{\gamma} u \mapsto \kappa c_{2}^{1 / 2} D_{y}^{1-\gamma} y^{\frac{a}{2}-1}=D_{y}^{1-\gamma} D_{y}^{\gamma} u=D_{y} u .
$$

$\mathrm{Al}$ evaluar la derivada fraccional de la potencia de la coordenada transversal, se tiene

$$
\kappa c_{2}^{1 / 2} \frac{\Gamma\left(\frac{a}{2}\right)}{\Gamma\left(\frac{a}{2}-1+\gamma\right)} y^{\frac{a}{2}-2+\gamma}=D_{y} u .
$$

Por lo que si $\frac{a}{2}-2+\gamma=-1$, se tiene $a=+2(1-\gamma)$. Pero, para que el esfuerzo exhiba un descenso con respecto a la coordenada transversal, tal como lo muestran los experimentos, se debe tener $\gamma>1$, [31]. Y, se recupera el modelo de Prandtl cuando $\gamma \rightarrow 1^{+}$. Pero también, se recupera el modelo de la ecuación (31), con el valor $\gamma=\frac{3}{2}$. Por tanto, el modelo de Prandtl se generaliza a un modelo fraccional que contiene los dos resultados: uno, el esfuerzo constante, la longitud de mezcla proporcional a la distancia transversal, y el perfil de velocidad logarítmico; y el otro, el esfuerzo decrece con la primera potencia de la distancia. Esta generalización da lugar a una interpretación probabilística, en sentido de que si el índice $\gamma \rightarrow 1^{+}$, podemos pensar en la interacción de los vórtices del tipo exponencial y un proceso de tipo de Poisson; pero en el caso de que este índice se aleja del valor unidad, pasamos a un proceso de Poisson de tipo fraccional, y el decaimiento exponencial se transforma en el decaimiento de la función Mittag-Leffler, en donde se alargan los tiempos de espera.

\subsection{Ecuación de Camassa-Holm}

El segundo caso tiene que ver con la ecuación viscosa de Camasa-Holm, cuyos detalles pueden consultarse en la referencia [5]. Pero, en particular, en su aplicación a los canales, y asumiendo turbulencia isótropa y homogénea, se reduce a una ecuación diferencial de orden 4, que puede reducirse aún más hasta el orden 2; y en donde el esfuerzo de Reynolds, en su componente 31, se expresa por la derivada de orden 3 de la velocidad, como en (41),

$$
\begin{gathered}
-\nu u^{\prime \prime}+\lambda^{2} u^{\prime \prime \prime \prime}=c t e_{1} \\
\tau_{31}=\nu \lambda^{2} u^{\prime \prime \prime}+p_{0} y+c t e_{2}
\end{gathered}
$$

en donde $u$ es la velocidad media; $\nu$ es la viscosidad cinemática; $\lambda$ es el parámetro de escala de longitud; $p_{0}$ es una constante; y, $y$ es la coordenada transversal al canal de ancho $2 y_{0}$, medida desde la línea media del canal. Con la condición de frontera en los bordes del canal $u\left( \pm y_{0}\right)=0$. La solución se expresa a través de dos constantes $c_{1}, c_{2}$ como una función complementaria al cosh que puede presentar un perfil lo suficientemente romo como para modelar el perfil turbulento,

$$
u(y)=c_{1}\left(1-\frac{\cosh \frac{y}{\lambda}}{\cosh \frac{y_{0}}{\lambda}}\right)+c_{2}\left(1-\left(\frac{y}{y_{0}}\right)^{2}\right) .
$$


Por tanto, el esfuerzo de Reynolds es

$$
\tau_{31}=-\frac{\nu}{\lambda} c_{1} \frac{\sinh \frac{y}{\lambda}}{\cosh \frac{y_{0}}{\lambda}}+p_{0} y+c t e .
$$

Este resultado, fruto de incrementar la derivada de orden entero hasta el orden 4 para la velocidad y hasta orden 3 para el esfuerzo, se consigue con la derivada de orden fraccional, porque la misma para (41), con $\lambda=1$, es

$$
\begin{aligned}
\tau= & -\left(e^{y} \frac{\gamma(\beta, y)}{2 \cosh y_{0} \Gamma(\beta)}+e^{-y} \frac{(-1)^{\beta} \gamma(\beta,-y)}{2 \cosh y_{0} \Gamma(\beta)}\right) \\
& +c_{2} \frac{2}{\Gamma(3-\beta)} y^{2-\beta}+\left(c_{1}+c_{2}\right) \frac{1}{\Gamma(1-\beta)} y^{-\beta}
\end{aligned}
$$

En donde $\gamma(\beta, y)$ y $\Gamma(\beta)$ son las funciones Gamma incompleta, inferior; y Gamma de Euler. Y, en el límite cuando $\beta \rightarrow 1$, la componente del esfuerzo, se reduce al resultado anterior (43), después de elegir, apropiadamente, las constantes;

$$
\tau_{31}=-c_{1} \frac{\sinh y}{\cosh y_{0}}+2 c_{2} y
$$

\section{La transición de fase}

El análisis de la transición de la velocidad lo podemos abordar también desde otro punto de vista. El reflejo de la figura 3 sobre la horizontal representa al esfuerzo con su signo incluido; y de ésta, puede destacarse la inyectividad de la función, lo que nos permite pensar en la función inversa $u(\tau)$, en donde a un valor del esfuerzo le corresponde un único valor de la velocidad; y se observa que cuando el esfuerzo crece, crece también la velocidad, por lo que es una función monótona creciente. Para el exponente $3-\beta \rightarrow 2^{+}$, (en las ecuaciones (33), (35), $(a=1)$ ), esta función inversa es cuadrática en el esfuerzo, pero si pensamos en una forma potencial, es posible definir la energía libre dependiente del esfuerzo dada en (46), cuya derivada reproduce la velocidad en términos del esfuerzo,

$$
E_{l}(\tau)=\frac{1}{2} u_{*} \frac{y_{0}}{l_{0}}\left(\tau-\frac{1}{3} \frac{\tau^{3}}{\tau_{0}^{2}}\right) .
$$

Desde el punto de vista termodinámico, la energía libre debe ser convexa, por lo que su segunda derivada, respecto del esfuerzo, ha de ser positiva, para que se representen los estados de equilibrio estables (observar que la variable $\tau$ es negativa). Entonces, y en el marco de la teoría de Landau, contamos con una energía libre de Helmholtz convexa; un parámetro de orden, el esfuerzo; y como respuesta energética del sistema a los cambios de este parámetro de orden, tenemos a la velocidad, función que debe ser creciente con respecto a dicho parámetro, [4].

El esfuerzo inercial es una función que, en magnitud, decrece con el crecimiento de la velocidad, que se anula en el plano de simetría que separa las placas, y es convexa. Para los valores numéricos citados, la gráfica se muestra en la figura 4. 
Reflejando la figura 4 sobre la horizontal, otra vez, destacamos la propiedad de la inyectividad de esta función, por lo que definimos la función inversa, en la que a cada valor del esfuerzo se le hace corresponder el único valor de la velocidad $u(\tau)$. Este reflejo nos muestra que cuando se incrementa el esfuerzo crece la velocidad, por lo que también es una función monótona creciente; y al introducir la forma potencial de esta función inversa, obtenemos la energía libre (47),

$$
E_{l}(\tau)=\frac{\kappa}{N^{2}} \tau_{0} u_{*}\left(-\frac{\tau}{\tau_{0}}+\frac{l}{y_{0}}\right)\left(\ln \left(\frac{1}{N}\left(-\frac{\tau}{\tau_{0}}+\frac{l}{y_{0}}\right)\right)-1\right)
$$

Ésta es una energía libre convexa del orden de magnitud cien veces menor que la mostrada en (46), para los valores numéricos que venimos ilustrando. Así que, contamos con otra energía libre, el mismo parámetro de orden y la misma respuesta energética del sistema.

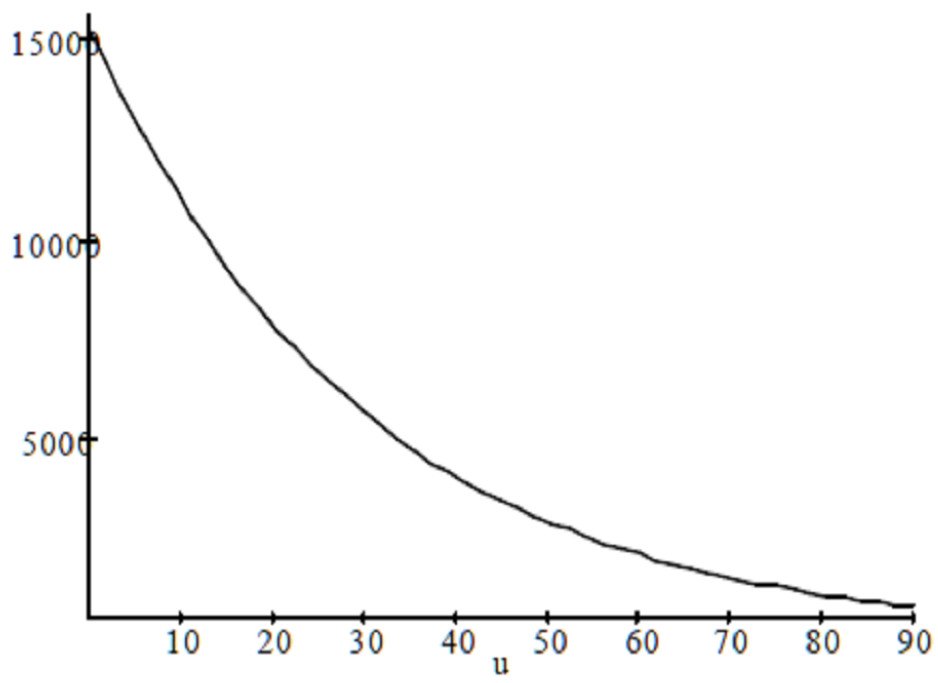

Figura 4: Esfuerzo inercial.

La condición de equilibrio debe corresponder al mínimo de la energía libre, por lo que es necesario agregar el término $-u(l) \tau$ a la energía libre, en donde a $u(l)$ la denominaremos, ahora, la velocidad de transición; de tal manera que la condición de equilibrio se exprese por la igualdad $u=u(l)$; lo que puede interpretarse como un ajuste del parámetro de forma, el esfuerzo, para que en el equilibrio corresponda al balance entre la acción externa, entendida como la acción de la pared, incluida la capa límite, sobre el fluido, y la respuesta interna, entendida como la acción del fluido sobre la capa límite. Por ejemplo, la primera energía libre queda como en (48), [4],

$$
E_{l}(\tau) \mapsto \tilde{E}_{l}(\tau)=E_{l}(\tau)-u(l) \tau=\frac{1}{2} u_{*} \frac{y_{0}}{l_{0}}\left(\tau-\frac{1}{3} \frac{\tau^{3}}{\tau_{0}^{2}}\right)-u(l) \tau .
$$

Por tanto, los esfuerzos de Reynolds sufren un salto de discontinuidad a la velocidad de transición, o a la distancia del espesor de la capa límite. De tal forma que, hasta la 
velocidad de transición mantiene un valor casi constante, para luego sufrir la transición, y finalmente, decaer exponencialmente hasta el plano de simetría; como se ilustra en la figura 5. Podemos todavía imaginar estados metaestables en donde las curvas de los esfuerzos de Reynolds se prolonguen un poco más allá de la línea vertical que hemos marcado como la transición, pero no son posibles estados que cierren la curva haciéndola continua, porque para ellos la respuesta del sistema no se comporta como función creciente.

Por tanto, describimos la transformación en el fluido como una transición de segundo orden, en donde el cambio ocurre en la simetría, sin haberlo en la fase líquida, en tanto estado de agregación de la materia. La transición de fase tiene dos energías libres convexas; como parámetro de orden, tenemos al esfuerzo de Reynolds, entendido como cantidad física que caracteriza la diferencia explícita entre las dos fases, y como respuesta energética del sistema, a la velocidad. Dicho cambio puede también interpretarse, a nivel microscópico, como un salto cualitativo en el índice de ocupación del espacio, de mayor a menor ocupación del mismo; el cual se describe como el salto en el valor del índice de ocupación desde un valor cercano a 1 hasta algún valor estrictamente menor que el anterior y cercano a 1/2. También, podemos decir que esta transición de segundo orden se describe como el salto desde la no-intermitencia a la intermitencia. Y finalmente, decimos que esta transición marca el salto desde la distribución gaussiana de índice Lévy 2, hasta alguna distribución Lévy de índice estrictamente menor que 2.

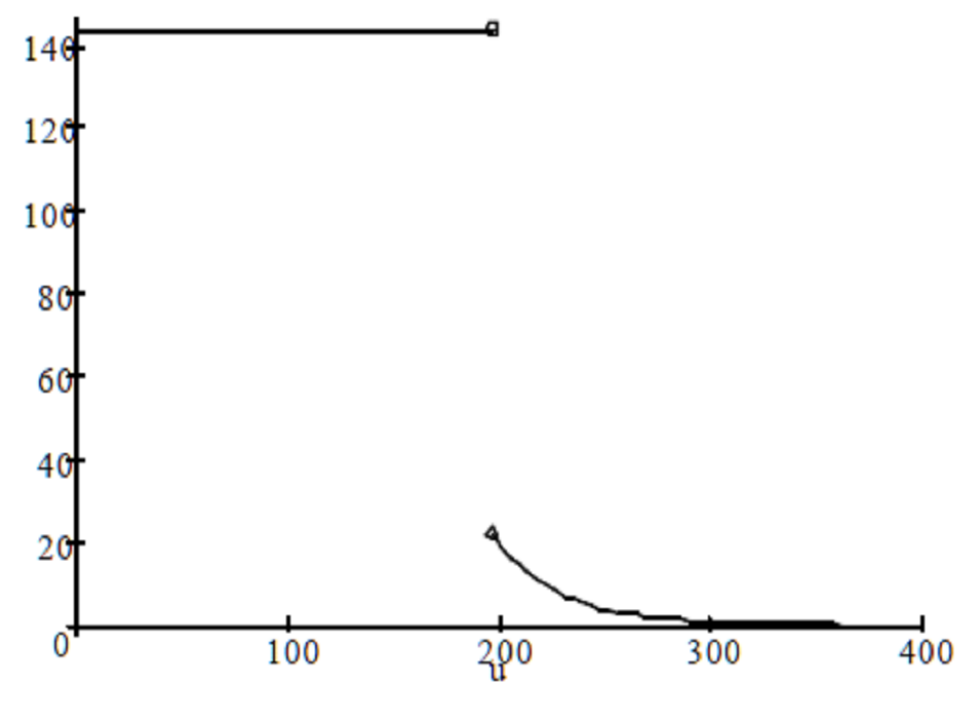

Figura 5: Esfuerzo inercial.

\section{Conclusiones}

- El esfuerzo de Reynolds es proporcional a la derivada fraccional de la velocidad media, siendo su orden, el índice de ocupación espacial. 
- Formulamos un modelo de Prandtl fraccional que muestra un descenso del esfuerzo con la coordenada transversal; pero que también permite recuperar su modelo tradicional.

- La constante de Nikuradse es el parámetro hidráulico, equivalente a la constante de Euler, que se expresa como la razón de las escalas laminar sobre la inercial; lo que posibilita a la misma constante de Euler ser interpretada como parámetro de escala.

- Las gráficas de los esfuerzos de Reynolds reproducen cualitativamente los resultados experimentales reportados por [31].

- Los resultados son compatibles con los obtenidos en [18], [21], como solución de un problema inverso para la función de reacción, con base en el análisis de las simetrías de la ecuación diferencial, que produjo formas analíticas para los esfuerzos de Reynolds, para el caso del flujo entre placas paralelas y en tubos cilíndricos, circulares, rectos.

- El fluido exhibe una transición de segundo orden, que se manifiesta con el salto de discontinuidad del esfuerzo de Reynolds, en tanto parámetro de orden, que se explica con una ruptura de la simetría, y sin ocurrir el cambio en el estado de agregación líquida de la materia.

- El salto a nivel microscópico se interpreta como la transición en la ocupación del espacio, de mayor a menor; o bien, para la subcapa viscosa se tiene un índice de ocupación cercano a la unidad; en tanto, para la subcapa inercial este índice es estrictamente menor que el anterior y cercano a $1 / 2$.

\section{Referencias}

[1] Ahmadi, G. (2005) "Review of turbulence", Clarkson U., (http://www.clarkson.edu/projects/crcd/me637).

[2] Arfken, G. (1985) Mathematical Methods for Physicists, 2nd ed. Academic, New York.

[3] Bluman, G.W.; Kumei, S. (1989) Symmetries and Differential Equations. SpringerVerlag, Berlin.

[4] Brokate M.; Sprekels, J. (1996) "Hysteresis and phase transitions", Applied Mathematical Sciences 121 Springer, New York, pp. 357.

[5] Chen, S.; Foias, C.; Holm, D.; Olson, E.; Titi, E.S.; Wynne, S. (1998) "Camasa-Holm equations as a closure model for turbulent channel and pipe flow", Physical Rev. Lett. 81(24) 5338-5341.

[6] Chen W., (2006) "A speculative study of 2/3-order fraccional Laplacian modeling of turbulence: Some thoughts and conjectures", Chaos 16, 023126. 
[7] Courant, R.; Hilbert, D. (1989) Methods of Mathematical Physics, Vol I. John Wiley \& Sons, New York.

[8] Doob J.L. (1960) Stochastic Processes. John Wiley \& Sons, New York.

[9] Fefferman, C.L. (2000) "Existence and smoothness of the Navier-Stokes equation", Princeton University, Department of Mathematics, Princeton, NJ08544-1000, May 1.

[10] Holmer, J. (2005) "The initial-boundary problem for the Korteweg-deVries equation", arXiv:math/0507168v1 [math.AP].

[11] Hörmander, L. (1983) The Analysis of Linear Partial Differential Operators I. Springer-Verlag, Berlin.

[12] Klewicki, J.; Fife, P.; Wei, T.; MacMurty, P. (2007) "A physical model of the turbulent boundary layer consonant with mean momentum balance structure", Phil. Trans. $R$. Soc. A 365: 823-839.

[13] Krasnov, M.; Kiseliov, A.; Makarenko, G. (1982) Ecuaciones Integrales. Editorial Mir, Moscú.

[14] Levi, E. (1989) El Agua Según la Ciencia. Conacyt, Ed. Castell Mexicana, México.

[15] Mainardi, F.; Pagnini, G.; Gorenflo, R. (2007) "Mellin transform and subordinate laws in fractional diffusion processes," arXiv:math/0702133v1 [math.PR].

[16] Meerschaert M; Mortensen, J.; Wheatcraft, S. (2005) "Fractional vector calculus for fractional advection-dispersion", Report University of Ontago, Dunedin 9001, New Zeland.

[17] Mercado J.R. (2008) "La ecuación de Navier-Stokes y multifractales", Revista de Matemática: Teoría y Aplicaciones 15(1): 49-72 .

[18] Mercado J.R. (2007) "Esfuerzos de Reynolds y simetrías", VIII Congreso Internacional de Ingeniería Hidráulica, ISBN 978-959-247-036-1. Isla de la Juventud, Cuba.

[19] Mercado, J.R.; Lázaro, P.; Brambila, F.; Fuentes, C. (2002) "Aproximación fractal para los semivariogramas de los niveles freáticos", Revista de Matemática: Teoría y Aplicaciones $\mathbf{9}(2)$.

[20] Mercado, J.R.; Brambila, F. (2001) "Problemas inversos en las ecuaciones de FokkerPlanck", Aportaciones Matemáticas, Serie Comunicaciones 29: 201-222.

[21] Mercado, J.R.; González, A.; Brambila, F. (2000). "Problemas inversos en las ecuaciones de Navier-Stokes", XVI Congreso Nacional de Hidráulica, Morelia, México: 1067-1072.

[22] Olver, P.J. (1993) Applications Lie Groups to Differential Equations. Springer-Verlag, Berlin. 
[23] Risken, H. (1989) The Fokker-Planck Equation. Springer-Verlag, Berlin.

[24] Rouse, H. (1946) Elementary Mechanics of Fluids Dover, New York.

[25] Sommerfeld, A. (1950) Mechanics of Deformable Bodies. Academic Press, New York.

[26] Sotelo, G. (1991) Hidráulica General, Vol. 1, Fundamentos. Noriega Limusa, México.

[27] Tarasov, V.E. (2007a) "Fractional derivative as fractional power of derivative", International Journal of Mathematics 18: 281-299.

[28] Tarasov, V.E. (2007b) "The fractional Chapman-Kolmogorov equation", Modern Physics Letters B 21: 163-174.

[29] Tarasov, V.E. (2005) "Fractional Fokker-Planck equation for fractal media", Chaos 15.

[30] Temam, R. (1984) Navier-Stokes Equations, Theory and Numerical Analysis. NorthHolland, Amsterdam.

[31] Wei, T.; Willmarth, W. (1989) "Reynolds-number effects on the structure of a turbulent channel flow", J. Fluid Mech. 204: 57-95. 